\title{
Dopamine acts through Cryptochrome to promote acute arousal in Drosophila
}

\author{
Shailesh Kumar, Dechun Chen, and Amita Sehgal ${ }^{1}$ \\ Howard Hughes Medical Institute, Department of Neuroscience, University of Pennsylvania School of Medicine, \\ Philadelphia, Pennsylvania 19104, USA
}

\begin{abstract}
The fruit fly, Drosophila melanogaster, is generally diurnal, but a few mutant strains, such as the circadian clock mutant $C_{1 k^{r k}}$, have been described as nocturnal. We report here that increased nighttime activity of Clk mutants is mediated by high levels of the circadian photoreceptor CRYPTOCHROME (CRY) in large ventral lateral neurons $\left(1-\mathrm{LN}_{\mathrm{v}} \mathrm{s}\right)$. We found that $\mathrm{CRY}$ expression is also required for nighttime activity in mutants that have high dopamine signaling. In fact, dopamine signaling is elevated in $C{ }^{J r k}$ mutants and acts through CRY to promote the nocturnal activity of this mutant. Notably, dopamine and CRY are required for acute arousal upon sensory stimulation. Because dopamine signaling and CRY levels are typically high at night, this may explain why a chronic increase in levels of these molecules produces sustained nighttime activity. We propose that CRY has a distinct role in acute responses to sensory stimuli: (1) circadian responses to light, as previously reported, and (2) noncircadian effects on arousal, as shown here.
\end{abstract}

[Keywords: cryptochrome; arousal; clock genes; dopamine; nocturnal/diurnal behavior]

Supplemental material is available for this article.

Received December 26, 2011; revised version accepted April 20, 2012.

Animals adapt to day:night cycles by selecting preferred times of sleep and wake. These preferred times, commonly referred to as temporal niches, optimize survival, most likely by permitting the best utilization of food resources or limiting exposure to predators. Contrary to popular belief, these temporal niches are not determined by the central circadian clock (Redlin and Mrosovsky 1999; Smale et al. 2003), although timekeeping by the clock helps the animal abide by its preferred niche. Nocturnal and diurnal mammals have the same clock mechanism in the central clock, the suprachiasmatic nucleus (SCN). In fact, the phase of cyclic clock gene expression is the same in the SCN in both types of animals (Smale et al. 2003).

Besides being a strong entraining cue for circadian rhythms, light promotes the activity of diurnal animals and suppresses the activity of nocturnal animals. In Drosophila, light-driven arousal involves a specific group of circadian neurons, called the large ventral lateral neurons (1-LN $\mathrm{L}_{\mathrm{v}}$ ) (Shang et al. 2008; Sheeba et al. 2008), which express canonical clock proteins but do not have a role in free-running circadian rhythms (rhythms in the absence of environmental cycles) (Nitabach and Taghert 2008). Consistent with light-driven activity being independent of circadian rhythms, loss of a functional

${ }^{1}$ Corresponding author.

E-mail amita@mail.med.upenn.edu.

Article published online ahead of print. Article and publication date are online at http://www.genesdev.org/cgi/doi/10.1101/gad.186338.111. circadian clock per se does not affect such activity. The Drosophila molecular clock consists of a transcriptionaltranslational feedback loop in which the CLOCK (CLK) and CYCLE (CYC) proteins activate transcription of the period (per) and timeless (tim) genes, and the PER and TIM proteins negatively regulate CLK-CYC activity (Zheng and Sehgal 2008). Mutants that lack the per or tim genes show loss of free-running rhythms under constant dark (DD) conditions but are still rhythmic and diurnal in the presence of light:dark (LD) cycles (Wheeler et al. 1993; Lacroix et al. 2004). Likewise, nocturnal mammals, such as hamsters, remain nocturnal in LD cycles even when they completely lack the central clock (Redlin and Mrosovsky 1999). However, $C l k^{\text {Irk }}$ flies, which are mutant for the Clk gene, display nocturnal behavior (Kim et al. 2002; Lu et al. 2008), suggesting that Clk has a noncircadian role in determining the Drosophila diurnal pattern. Interestingly, Clk and cyc mutants also show clock-independent reductions in total sleep time, suggesting that they regulate arousal (Hendricks et al. 2003). However, the mechanisms underlying the switch to nocturnal behavior are not known, nor is it known whether the arousal phenotype is related to the nocturnal behavior.

We sought to address the molecular and neural basis of the nocturnal behavior of $C l k^{I r k}$ flies. We report here that levels of the circadian photoreceptor CRYPTOCHROME (CRY) are elevated in Clk mutants and act in $1-\mathrm{LN}_{\mathrm{v}} \mathrm{s}$ to drive nighttime activity. CRY is also required for increased night activity of mutants that have increased dopamine 
signaling. Based on these findings, we investigated dopamine signaling in Clk mutants and found that it is significantly high and is responsible for the nocturnal behavior and, most likely, also for the increased arousal. Finally, we report that the night activity-promoting effect of dopamine and CRY reflects a normal role of these molecules in mediating acute responses of the animal to sensory stimuli.

\section{Results}

Nocturnal activity of $\mathrm{Clk}^{\mathrm{Jk}}$ mutants is mediated by elevated CRY

The original $C l k^{I r k}$ mutants display nocturnal behavior in the presence of LD cycles (Kim et al. 2002; Lu et al. 2008). They were also shown to express high levels of the circadian photoreceptor CRY (Emery et al. 1998). Since neither of these phenotypes is observed in other circadian clock mutants (Wheeler et al. 1993; data not shown), and the nocturnal behavior reflects an aberrant response to photic stimuli, we asked whether the two effects are related. We first confirmed these phenotypes of $C 1 k^{I r k}$ by outcrossing the mutant allele for seven generations into an isogenic wild-type background (Iso31) used specifically for behavioral experiments (Koh et al. 2008). As shown in Figure 1A, CRY levels are substantially higher in $C l k^{J r k}$ flies during the dark phase (Fig. 1A, zeitgeber time 14 [ZT14] and ZT20) of the LD cycle, although they are still cyclic, indicating that CRY is still degraded by light (Lin et al. 2001). Also, the outcrossed Clk ${ }^{\text {Irk }}$ flies still display nocturnal behavior (Fig. 1B). $c y c^{01}$ mutants also show higher nighttime activity in LD cycles (Supplemental Fig. 1A).

To determine whether the high levels of CRY account for the noncircadian phenotype (i.e., the high nighttime activity) of $C l k^{\text {Irk }}$ mutants, we generated double mutants of $C l k^{I r k}$ and $c r y{ }^{b}$. Upon testing the $C l k^{I r k}{ }^{k},{ }^{2} y^{b}$ double mutants, we found that nighttime activity was significantly reduced as compared with that in Clk ${ }^{I r k}$ mutants (Fig. 1B; Supplemental Fig. 1B). To obtain a more quantitative measure of the temporal preference of these animals, we calculated a diurnal/nocturnal index (see the legend for Fig. 1), which indicated nocturnal behavior of $C l k^{I r k}$ flies but lack of a time-of-day preference in $C k^{\text {Irk }}{ }^{\text {cry }}{ }^{b}$ mutants. The $c^{2}{ }^{b}$ mutation is a point mutation in the flavin-binding region of CRY. It eliminates photosensitivity of CRY and also greatly reduces levels of the protein (Stanewsky et al. 1998); however, it is not a null mutation. To test the effects of a cry-null mutation, we recombined a $c r y^{02}$ allele with $C l k^{I r k}$ and assayed the behavior of the resulting double mutants. The nocturnal phenotype of $C l k^{I r k}$ flies was completely suppressed by $\mathrm{cry}^{02}$, such that the double mutants displayed strong diurnal behavior similar to that of wildtype Iso31 controls and cry $^{02}$ flies (Fig. 1B). To determine whether the suppression of nocturnal behavior was due to a decrease in nighttime activity or an increase in daytime activity, we measured activity levels in single and double mutants and found that the cry mutations reduced nighttime activity (Supplemental Fig. 1B). The cry ${ }^{02} \mathrm{mu}-$ tation also reduced daytime activity, although less so than nighttime, but in neither case did the suppression of nocturnal behavior result from increased daytime activity. These data indicate that the high nighttime activity of $C l k^{\text {Irk }}$ flies is due to higher CRY levels.

\section{High levels of CRY in 1-LN $N_{V}$ contribute to the nocturnal activity phenotype of $\mathrm{Clk}^{\text {Jrk }}$ flies}

To determine where in the fly brain CRY is required in $C l k^{I r k}$ flies to promote nocturnal activity, we reintroduced
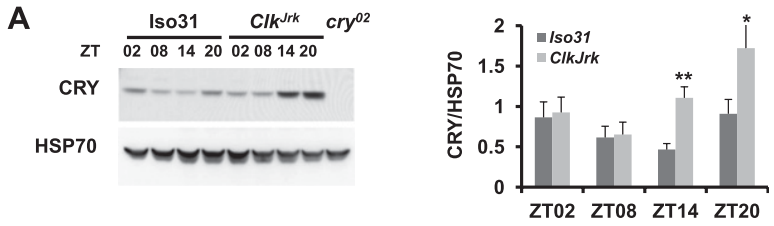

B
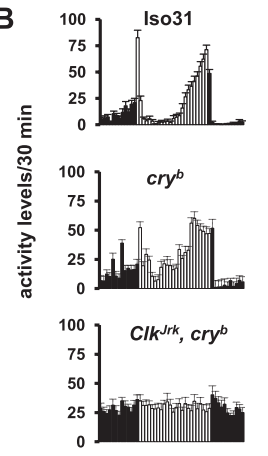
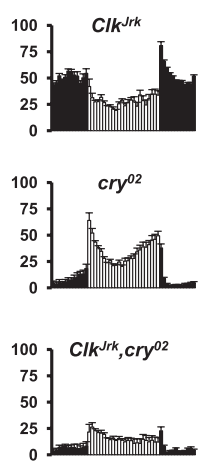

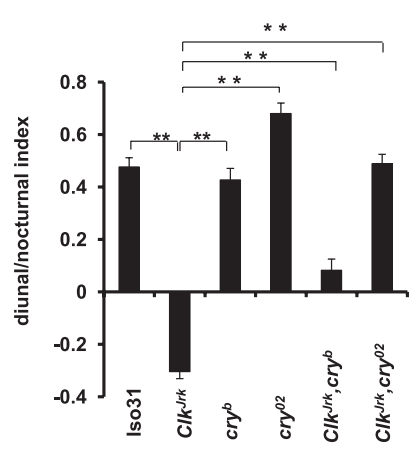

Figure 1. Nighttime activity-promoting effects of the $C_{1 k^{r k k}}$ mutation require CRY. (A) Levels of CRY are high in $C_{1 k}{ }^{I r k}$ flies. CRY levels were assayed in adult heads of Iso31 and $C l k^{r k}$ flies through Western blot analysis. HSP70 antibodies were used to control for loading. The right panel shows the average CRY levels in Iso31 and $C l k^{r r k}$ flies assayed in five independent experiments. CRY levels are significantly higher in the Clk ${ }^{\text {Irk }} \mathrm{mu}$ tants than in Iso31 flies during the dark phase of the LD cycle. $\left(^{\star}\right) P<0.05 ;\left({ }^{\star \star}\right) P<0.01$. Error bars indicate the SEM. (B, left panel) Pattern of activity in Iso31 controls $(n=16)$, Clk $^{\text {Irk }}(n=48)$, cry $^{b}(n=31)$, cry $^{02}(n=31)$,

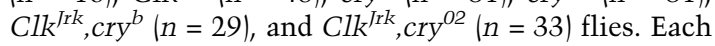
panel depicts the average daily locomotor activity, starting at ZT18, for a given genotype. The records are based on activity data from three consecutive 24-h periods. Vertical bars represent activity recorded in 30min bins during times when the lights were either on (white bars) or off (black bars). (Right panel) Diurnal/ nocturnal index for the lines shown in $A$ and the left panel of $B$. This was calculated as (total activity during the day) - (total activity during the night)/(total activity), averaged over a 3-d period per fly. Preference for activity during the day is represented as positive values, and preference for activity during the night is shown as negative values for each genotype. Asterisks above bars denote significant differences between genotypes. $\left.\left(^{\star}\right) P<0.05 ;{ }^{\star \star}\right) P<0.01$. Error bars depict SEM and indicate variability across flies of a specific genotype. The significance level is shown for pairwise comparisons as indicated. 
CRY into $C 1 k^{I r k}$, cry $^{b}$ and $C l k^{J r k},{ }^{2}{ }^{02}{ }^{02}$ double mutants. The idea was to determine whether nocturnal activity could be restored by overexpression of CRY in specific cells. UAS-CRY and different GAL4 transgenes were crossed into each of the double-mutant backgrounds, and flies were tested for behavior under LD conditions (Fig. 2A,B; Supplemental Fig. 2A,B). Similar results were obtained with both mutant backgrounds, although there were small differences in the magnitude of the effect. One of the most effective drivers in increasing nocturnal behavior of $C l k^{\text {Irk }}$,cry double-mutant flies was per $^{1 b}$ GAL4, which is selectively expressed in a subset of clock neurons: the 1- $\mathrm{LN}_{\mathrm{v}} \mathrm{s}$ and some dorsal neurons (all DN1s and a few DN2 and DN3 clock neurons) (Kaneko and Hall 2000). Increased nighttime activity with this driver is evident from the histogram shown in Figure 2, A and B, and is also indicated by the diurnal-nocturnal index (Fig. 2A,B). The gmr-GAL4 driver expressing CRY in all photoreceptor cells did not restore the $C l k^{\text {Jrk }}$ LD nocturnal phenotype. On the other hand, CRY expression driven by tim27-GAL4, which expresses in all clock cells, resulted in a nocturnal preference. Expression of CRY by $P d f$-GAL4, which is expressed in only the small $\mathrm{LN}_{\mathrm{v}} \mathrm{s}$
(s-LN $\left.\mathrm{L}_{\mathrm{v}} \mathrm{s}\right)$ and $1-\mathrm{LN}_{\mathrm{v}} \mathrm{s}$, also resulted in a small shift from daytime to nighttime activity, but less so than with per $^{1 b}$ and tim27, perhaps because it is a weaker driver. To further delineate the relevant neurons, we used the c929GAL4 driver. c929-GAL4 is expressed in all peptidergic neurons, including the $1-\mathrm{LN}_{\mathrm{v}} \mathrm{s}$, and the latter happen to be the only clock cells labeled by this driver (Taghert et al. 2001). We found that expression of CRY by c929 in the double-mutant background also increased the nocturnal preference of $\mathrm{Clk}^{\mathrm{Irk}}{ }^{,} \mathrm{cry}^{b}$ and, even more so, $\mathrm{Clk}^{\mathrm{Irk}}, \mathrm{cry}^{\mathrm{O}}$ flies (Fig. 2B; Supplemental Fig. 2A,B). Given that the only area of overlap between all of these different drivers is the $1-\mathrm{LN}_{\mathrm{v}} \mathrm{s}$, we surmise that CRY expression in these cells is necessary to drive the nocturnal phenotype of Clk mutants. As noted above, the $1-\mathrm{LN}_{\mathrm{v}} \mathrm{s}$ typically promote arousal in response to light, so it is not surprising that the CRY-mediated switch to nocturnal activity also occurs in these cells.

To test whether CRY overexpression is sufficient to produce nocturnal behavior, we overexpressed CRY in a wild-type background using various clock neuron drivers (Pdf, c929, per $^{1 b}$, and tim27) and eye-specific gmr drivers; however, they did not affect the day:night distribution of
A

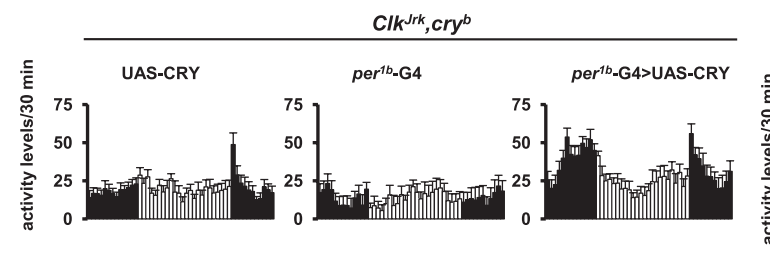

B
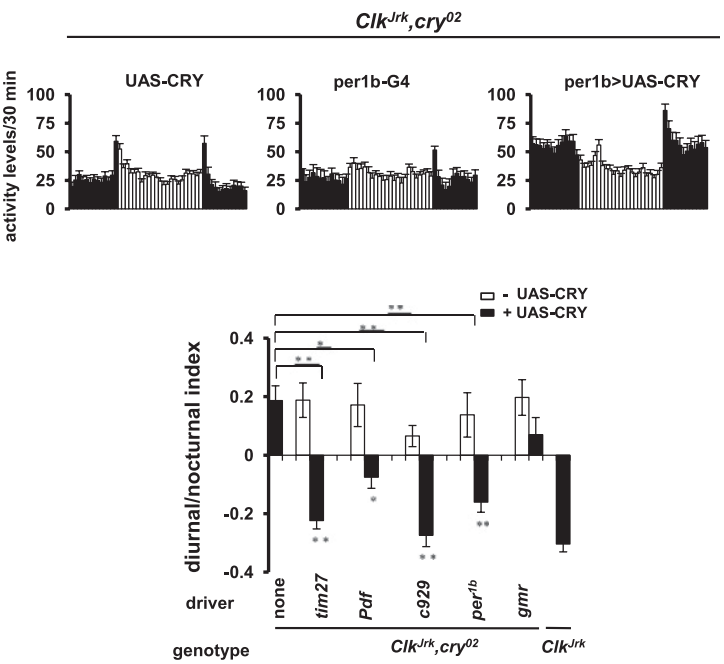

Figure 2. CRY expression in large $\mathrm{LN}_{\mathrm{v}} \mathrm{s}$ drives nocturnal behavior in Clk mutant flies. CRY was re-expressed in subsets of neurons in $\mathrm{Clk}^{I r k}$, cry $^{b}(A)$ and $\mathrm{Clk}^{I r k}$, cry $^{02}(B)$ flies to identify those in which it is required for nocturnal behavior. $(A$, top panel) Representative histograms of activity patterns are depicted for the driver that is most restricted to $1-\mathrm{LN}_{\mathrm{v}} \mathrm{s}-\mathrm{per}^{1 b}$-GAL4. Control flies (UAS-CRY;

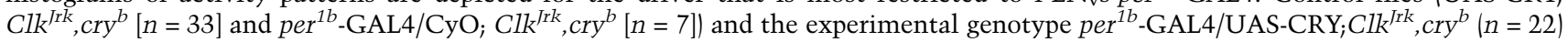
are depicted. As in Figure 1, each panel depicts the average daily locomotor activity based on data recorded over three consecutive 24-h periods. Vertical bars represent activity recorded in 30-min bins during times when the lights were either on (white bars) or off (black bars). (Bottom panel) Nocturnal/diurnal preference in Clk ${ }^{r k k}$, cry ${ }^{b}$ flies expressing CRY in different sets of neurons. Nocturnal/diurnal preference was calculated as described in Figure 1. The diurnal/nocturnal indices of tim27, c929, and per ${ }^{1 b}$ GAL4 driving UAS-CRY in a $C l k^{r r k}, c_{r y}^{b}$ background were significantly different from those of GAL4 and UAS-CRY controls (histograms for all other genotypes are shown in Supplemental Fig. 1A). However, the diurnal/nocturnal index of Pdf-GAL4/UAS-CRY flies was only marginally different from that of GAL4 and UAS-CRY controls $(P>0.05)$. $(B$, top panel) Representative histograms of activity patterns are depicted for control flies (UAS-CRY; Clk ${ }^{r k k}, c^{02}{ }^{02}[n=32]$ and per $^{1 b}$-GAL4/CyO; Clk $^{\text {rrk }}$, cry $^{02}[n=14]$ ) and the experimental genotype per ${ }^{1 b}$-GAL4/UAS-

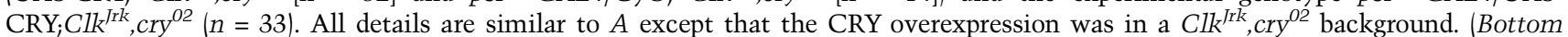
panel) Nocturnal/diurnal preference in $C l k^{I r k}, c r y{ }^{02}$ flies expressing CRY in different sets of neurons. The diurnal/nocturnal indices of tim27, Pdf-GAL4, c929, and per $^{1 b}$ GAL4 driving UAS-CRY in a Clk ${ }^{r k}$, cry ${ }^{02}$ background were significantly different from those of GAL4 and UAS-CRY controls. The $X$-axis denotes the genotype, whereas the $Y$-axis denotes whether the animals are diurnal (positive values) or nocturnal (negative values). $\left(^{\star}\right) P<0.05 ;\left(^{\star \star}\right) P<0.01$. Error bars indicate the SEM. 
activity (data not shown). Since $C l k^{I r k}$ lacks a functional circadian clock, we reasoned that high CRY might produce nocturnal behavior only in a genetic background lacking a functional circadian clock. Thus, we overexpressed CRY in a per-null $\left(\right.$ per $\left.^{O}\right)$ background. We found that the effect was a little stronger than that seen in wild type but did not result in nocturnal behavior (data not shown). These data indicate that overexpression of CRY alone is necessary but not sufficient to promote nocturnal behavior in $C l k^{\text {Irk }}$ flies.

\section{Elevated CRY levels contribute to the high nighttime activity in fumin (fmn) mutants}

Interestingly, $C l k^{I r k}$ flies are not the only ones that show increased nighttime activity under LD conditions. A dopamine transporter mutant, fmn, which has elevated dopamine signaling, also shows heightened nighttime activity in LD cycles. Unlike $C l k^{\text {Irk }}$ flies, fmn mutants are rhythmic in DD (see Supplemental Table 1) but also have an increased arousal phenotype (Kume et al. 2005). We confirmed the increased nighttime activity in $\mathrm{fmn}$ mutants (Fig. 3) and then asked whether this increase was also mediated by CRY. To determine whether fmn affects CRY levels, we measured CRY expression in adult heads through Western blots. The fmn flies, like $C l k^{I r k}$ mutants, exhibited significantly higher levels of CRY than Iso31 controls, particularly in the dark phase of the LD cycle (Fig. 3A). The higher levels of CRY in fmn mutants are likely due to post-transcriptional regulation, as the expression of cry mRNA is unaltered; it is expressed cyclically at levels comparable with those in wild type
(Supplemental Fig. 3). On the other hand, Clk ${ }^{I r k}$ mutants express noncycling and peak levels of cry mRNA throughout the LD cycle. The effect on the mRNA is likely due to decreased expression of the Clk target vri, which is a repressor of cry (Cyran et al. 2003). Thus, in Clk mutants, the effects of increased dopamine on CRY are coupled with increases in cry mRNA to produce very high levels of CRY.

To determine whether the increased nighttime activity phenotype of $f m n$ is due to elevated levels of CRY, we created double mutants of fmn with cry $^{b}\left(f_{m n} ; c^{\prime} y^{b}\right)$ and cry $^{02}\left(\mathrm{fmn}_{\mathrm{i}} \mathrm{cry}^{\mathrm{O}}\right)$, respectively. We found that nighttime activity was significantly reduced in both double mutants such that these mutants showed a diurnal preference similar to that of Iso31, cry $^{b}$, or cry ${ }^{02}$ flies (Fig. 3B). Thus, the heightened nighttime activity phenotype of fmn mutants is mediated by CRY.

\section{Dopamine levels are increased in $\mathrm{Clk}^{\mathrm{Jrk}}$ mutants}

Based on the effects of $f m n$ on nighttime activity and CRY, we investigated the possibility that the Clk mutation was also promoting nighttime activity through increases in dopamine. Thus, we assayed transcript levels of ple, the Drosophila gene encoding tyrosine hydroxylase (TH), a rate-limiting enzyme important for synthesis of L-DOPA (a precursor of dopamine) from tyrosine (FriggiGrelin et al. 2003). Specifically, we focused on the shorter ple-PA isoform, which is expressed only in the CNS (Friggi-Grelin et al. 2003). We found that levels of ple$P A$ mRNA cycle robustly in wild-type Iso31 flies, with a peak in the early morning (ZTO2) and a trough at ZT14
A
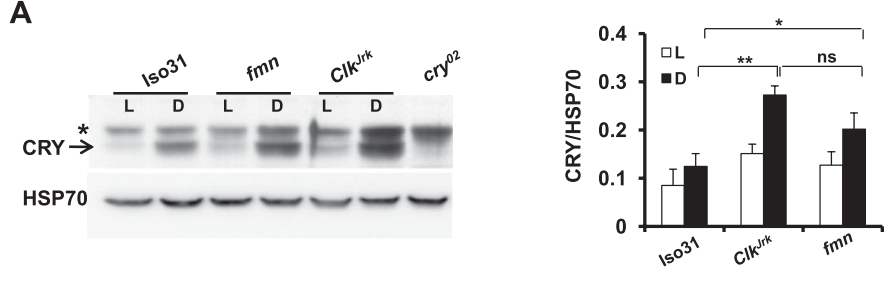

B

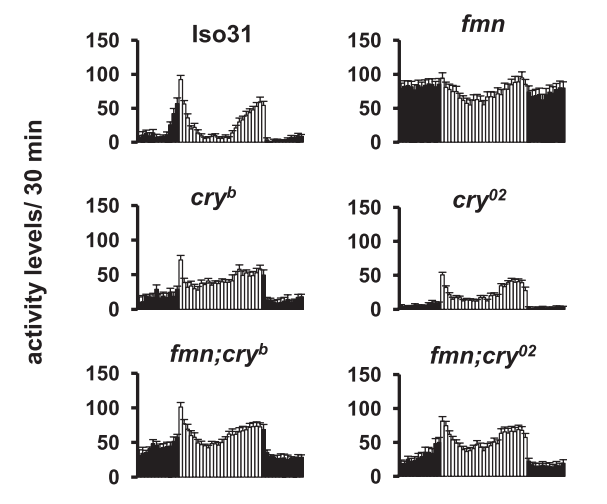

Figure 3. CRY mediates increased nighttime activity in flies with increased dopamine. (A, left panel) CRY expression in Iso31, Clk ${ }^{I r k}$, and fmn flies during the light (ZT08) and dark (ZT20) phases of an LD cycle. A representative Western blot is shown. CRY levels cycle in all genotypes indicated, but are significantly higher in the $C k^{I r k}$ and $f m n$ mutants than in Iso31 flies during the dark phase of the LD cycle. HSP70 antibodies are used to control for loading. The asterisk shows a nonspecific band in all lanes. (Right panel) Quantification of four independent experiments shows significantly increased CRY levels during the dark in $C_{1 k}{ }^{I r k}$ and $f m n$ flies compared with those in the wild-type Iso31 controls. Asterisks above the bars denote significant differences between genotypes. $\left(^{*}\right) P<0.05$; $\left(^{\star \star}\right) P<0.01$. Error bars depict SEM. $(B)$ Suppression of the nocturnal behavior of fmn through a reduction of CRY. (Left panel) Pattern of activity in Iso31 controls $(n=12)$, fmn $(n=30)$, cry $^{b}(n=12)$, cry $^{02}(n=$ $34), f^{2} n ; c r y{ }^{b}(n=66)$, and fmn;cry ${ }^{02}(n=30)$ flies. The records are based on activity data from four consecutive 24-h periods. Other details are similar to those in Figures 1 and 2. Asterisks above bars denote significant differences between genotypes. $\left(^{\star}\right) P<0.05 ;\left(^{\star \star}\right) P<0.01$. Error bars depict SEM and indicate variability across flies of a specific genotype. 
A

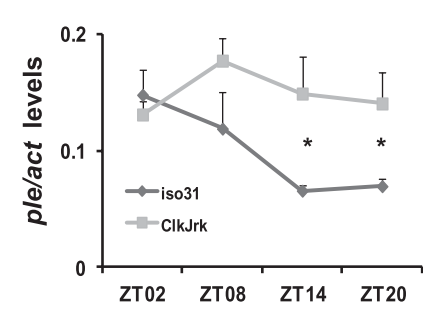

B

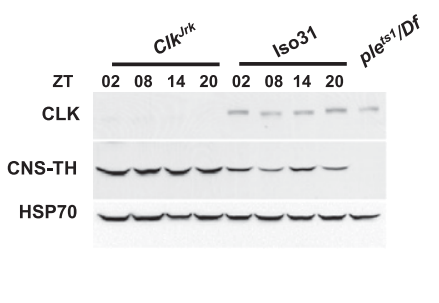

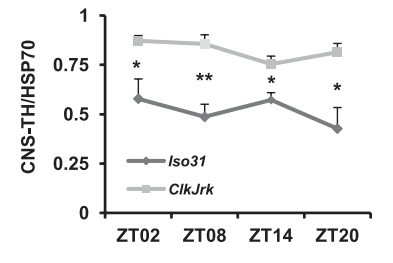

C
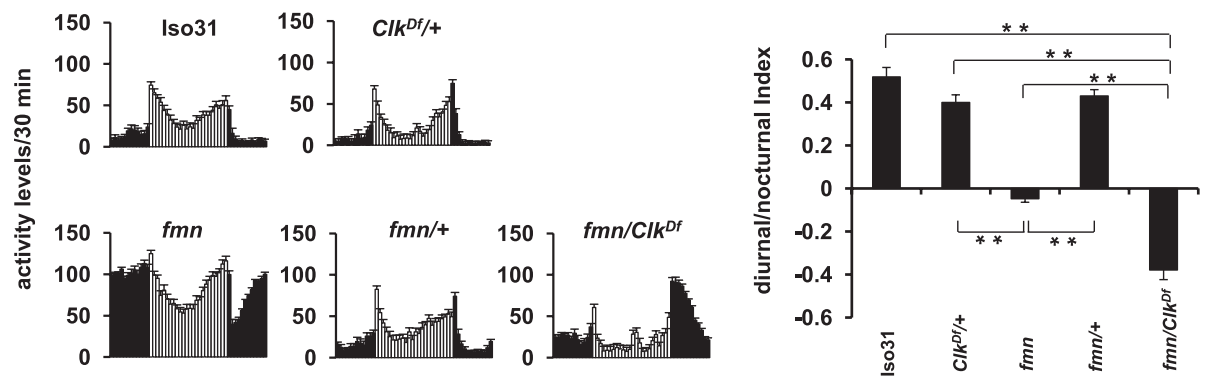

Figure 4. Altered dopamine levels in $C l k^{I r k}$ mutants contribute to nocturnal behavior. (A) Expression of the CNS-specific isoform of TH (ple-PA) mRNA in Iso31 and Clk ${ }^{r k}$ flies maintained in LD cycles. mRNA levels were measured in $\sim 30$ heads for each genotype by quantitative PCR. The $C l k^{r k}$ flies exhibit significantly higher levels of ple mRNA than do Iso31 control flies. Data were pooled from three independent experiments. In all experiments, the ple-PA mRNA levels were normalized to actin5C mRNA levels. $(B)$ Expression of the TH protein in heads of Iso31 and Clk ${ }^{r k k}$ flies under LD conditions. The CNS-specific isoform of TH is significantly reduced in mutant $\left[p l e^{t s 1} / D f(3 L) v n^{65 c}\right]$ flies. Clk ${ }^{\mid r k}$ mutants show higher levels of TH than Iso31 controls (see quantification on the right). Asterisks denote significant differences between genotypes. $\left.\left(^{*}\right) P<0.05 ;{ }^{* \star}\right) P<0.01$. Error bars depict SEM. $(C)$ Synergistic effects of the dopamine transporter mutant $\mathrm{fmn}$ and a Clk deficiency in promoting nocturnal activity. (Left panel) The average activity pattern of the indicated genotypes in LD cycles. Diurnal/nocturnal indices are indicated on the right. Iso31 $(n=16)$ and heterozygotes $C l k^{D f} /+(n=30)$ and $f m n /+(n=16)$ are diurnal, whereas homozygotes $f m n(n=14)$ or transheterozygotes of $f m n / C l k^{D f}(3 \mathrm{~L})^{R M 5-2}(n=30)$ display nocturnal activity. The records are based on activity data from four consecutive 24-h periods. Other details are similar to those in Figures 1-3. Asterisks above bars denote significant differences between genotypes. $\left.\left(^{\star}\right) P<0.05 ;{ }^{\star \star}\right) P<0.01$. Error bars indicate the SEM and indicate variability across flies of a specific genotype.

(Fig. 4A), but remain at peak levels throughout the LD cycle in $\mathrm{Clk}^{\text {rk }}$ flies (Fig. 4A). Next, we examined levels of $\mathrm{TH}$ in the heads of Iso31 and $C \mathrm{lk}^{I r k}$ flies through Western blots. Levels of the CNS-specific isoform of TH were significantly higher in Clk ${ }^{r k k}$ mutants than in Iso31 controls at all times of day (Fig. 4B). The increased TH levels in $C l k^{\text {Irk }}$ flies supports the idea that elevated dopamine signaling leads to the nocturnal phenotype of Jrk mutants.

To determine whether the elevated dopamine contributes to the nocturnal phenotype of $C l k^{j r k}$ mutants, we first looked for genetic interactions between Clk and $\mathrm{fmn}$. Flies heterozygous for $\mathrm{fmn}(\mathrm{fmn} / \mathrm{H})$ showed a normal diurnal pattern of activity (Fig. 4C). Since the $\mathrm{Clk}^{I r k}$ mutation has a semidominant effect on nocturnal activity (data not shown), we focused on flies heterozygous for the Clk deficiency $\left(C l k^{D f}\right)$. Flies heterozygous for the Clk deficiency were diurnal (Fig. 4C); however, transheterozygotes carrying a single copy of fmn together with a single copy of $C l k^{D f}$ displayed a strong nocturnal phenotype, suggesting synergy between loss of CLK and increased dopamine signaling (Fig. 4C). The increased TH levels and synergistic effects of $C l k^{D f}$ and fmn mutations on nighttime activity suggest that CLK activity normally regulates diurnal behavior through effects on the dopaminergic system.

\section{Increased dopamine signaling acts through CRY} to drive nocturnal behavior of Clk mutants

We next determined whether blocking dopamine release from TH-positive neurons could rescue the nocturnal phenotype of $\mathrm{Clk}^{I r k}$ mutants. We expressed either a modified potassium channel (UAS-Eko) or the tetanus toxin light chain (UAS-Tnt) in dopaminergic cells using the TH-GAL4 driver. Eko is a noninactivating form of the Shaker $\mathrm{K}^{+}$channel that inhibits neural activity of cells that express it (White et al. 2001), while Tnt blocks evoked exocytosis at fast synapses (Sweeney et al. 1995). Either transgene is expected to inhibit neurotransmission. Adult flies expressing Tnt or Eko in dopaminergic neurons in a wild-type background were viable and showed normal distribution of activity under LD conditions (Fig. 5A; Supplemental Fig. 4). Interestingly, when either of these two transgenes was expressed by the THGAL4 driver in the $C l k^{I r k}$ mutant background, nocturnal behavior was significantly suppressed, although the profile of activity did not revert from nocturnal to diurnal, 
A
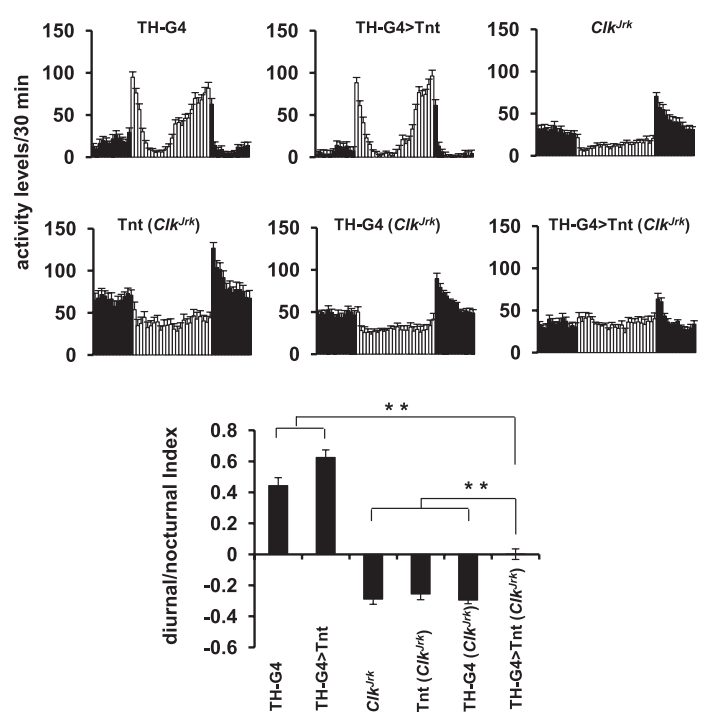

B
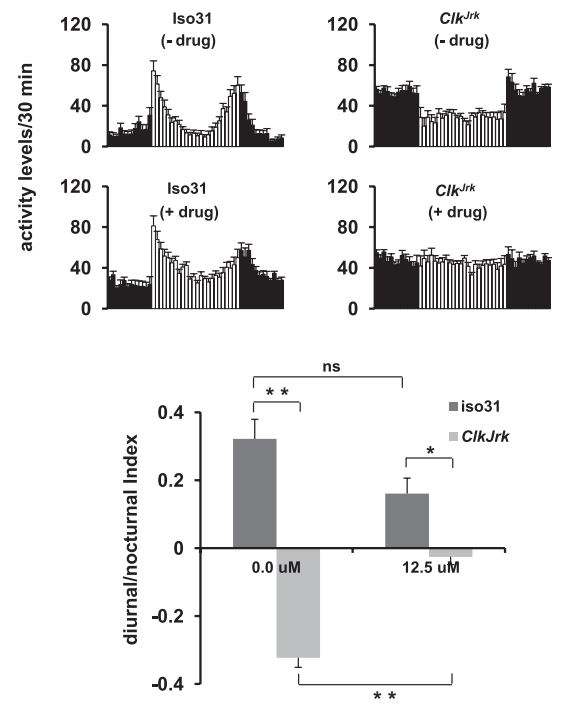

Figure 5. Blocking dopamine (DA) signaling rescues the nocturnal phenotype of $C l k^{I r k}$ mutants. (A) Overexpression of the UAS-Tnt transgene in dopaminergic neurons by a TH-GAL4 driver in a wild-type background does not affect the activity-rest pattern, but in a $C l k^{\text {rk }}$ genetic background, it leads to significant suppression of nighttime activity. (Top panel) Average activity patterns are shown for the following genotypes: TH-GAL4 control $(n=16)$, TH-GAL4>UAS-Tnt in a wild-type background $(n=11), C 1 k^{I r k}(n=29)$, UAS-Tnt control $(n=22)$, TH-GAL4,Clk ${ }^{r k k}$ control $(n=44)$, and TH-GAL4> UAS-Tnt in a Clk ${ }^{\text {Irk }}$ background $(n=26)$. (Bottom panel) Diurnal/ nocturnal indices of the genotypes indicated. The records are based on activity data from four consecutive 24-h periods. $(B)$ Rest:activity patterns of Iso31 and Clk ${ }^{\text {Irk }}$ mutants exposed to food containing haloperidol $(12.5 \mu \mathrm{M})$. Average activity patterns are indicated for the following genotypes: Iso31 $(n=17)$ and $C l k^{r r k}$ mutants $(n=27)$ in normal food with DMSO, and Iso31 $(n=15)$ and $C l k^{I r k}$ mutants $(n=15)$ in food containing drug. The diurnal/nocturnal indices on the bottom show that nighttime activity was significantly reduced in $C l k^{I r k}$ mutants treated with $12.5 \mu \mathrm{M}$ haloperidol but not in Iso31 flies. The records are based on activity data from four consecutive 24-h periods. Other details are similar to those in Figures 1-3. Asterisks above bars denote significant differences between genotypes. $\left({ }^{\star}\right) P<$ $0.05 ;\left(^{\star \star}\right) P<0.01$. Error bars indicate the SEM and indicate variability across flies of a specific genotype.

perhaps because dopamine release was not completely blocked (Fig. 5A; Supplemental Fig. 4). We also addressed the basis of the suppression by determining total activity levels during the day and night. We found that nighttime activity levels were significantly reduced in flies in which dopamine release was blocked, whereas the daytime activity levels remained largely unaffected (Supplemental Fig. 5A).

We also tested whether administering a dopamine receptor antagonist could rescue the nocturnal phenotype of $C l k^{r r k}$ flies. Haloperidol is an anti-psychotic drug that blocks dopamine receptors in humans and is also effective in insects (Blenau and Baumann 2001). When exposed to $12.5 \mu \mathrm{M}$ haloperidol in food, $C l k^{J r k}$ mutants showed a significant suppression of nighttime activity (Fig. 5B), whereas the same dose of drug did not alter rest:activity behavior or the diurnal/nocturnal index of Iso31 controls (Fig. 5B). At higher doses such as $25.0 \mu \mathrm{M}$ and $50.0 \mu \mathrm{M}$, the majority $(\sim 60 \%-80 \%)$ of Iso31 flies were dead or sick, whereas $\sim 70 \%-90 \%$ of the $C l k^{I r k}$ flies showed rest:activity behavior similar to those treated with $12.5 \mu \mathrm{M}$ (data not shown). As with the genetic block of dopamine, the pharmacological block also significantly reduced nighttime activity in $C l k^{I r k}$ mutants (Supplemental Fig. 5B). On the other hand, Iso31 flies showed no significant change in their daytime or nighttime activity levels (Supplemental Fig. 5).
To confirm that the elevated dopamine signaling in Clk mutants contributes to the high CRY, we examined CRY levels in $C l k^{I r k}$ mutants in which dopamine release was blocked by expressing UAS-Tnt in TH-positive cells. We found that CRY levels were significantly reduced during the dark phase in Clk ${ }^{\text {Irk }}$ flies under these conditions (Fig. $6 \mathrm{~A})$. We conclude that loss of Clk leads to increased expression of $\mathrm{TH}$ and dopamine, which in turn acts through CRY in the $1-\mathrm{LN}_{\mathrm{v}} \mathrm{s}$ to promote nocturnal activity.

As noted above, overexpression of CRY was necessary but not sufficient to produce nocturnal behavior. To determine whether elevated dopamine was sufficient to promote night activity, we overexpressed a bacterial sodium channel transgene (UAS-NachBac) in dopaminergic neurons of wild-type flies using a TH-GAL4 driver. Flies expressing NachBac in TH-positive cells showed significantly increased nighttime activity as compared with TH-GAL4 and NachBac controls (Fig. 6B). We further tested a requirement for CRY in this phenotype by expressing NachBac in dopaminergic neurons in a cry ${ }^{02}$ background. Loss of CRY significantly reduced the high nighttime activity of flies with increased dopamine signaling (Supplemental Fig. 6).

Dopamine and CRY are required for startle responses to sensory stimulation at night

Our data indicate that increased dopamine and CRY promote activity at night. Since flies typically sleep much 

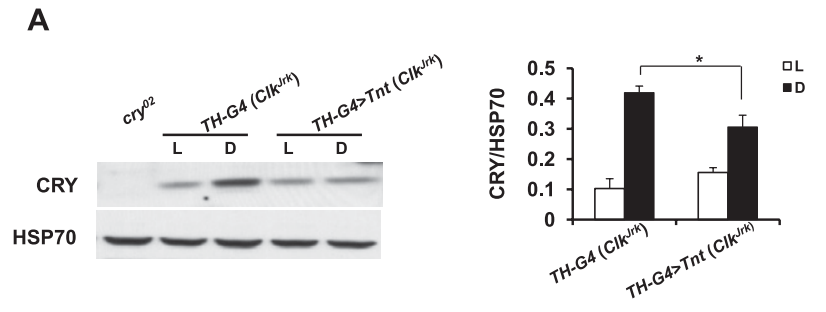

B
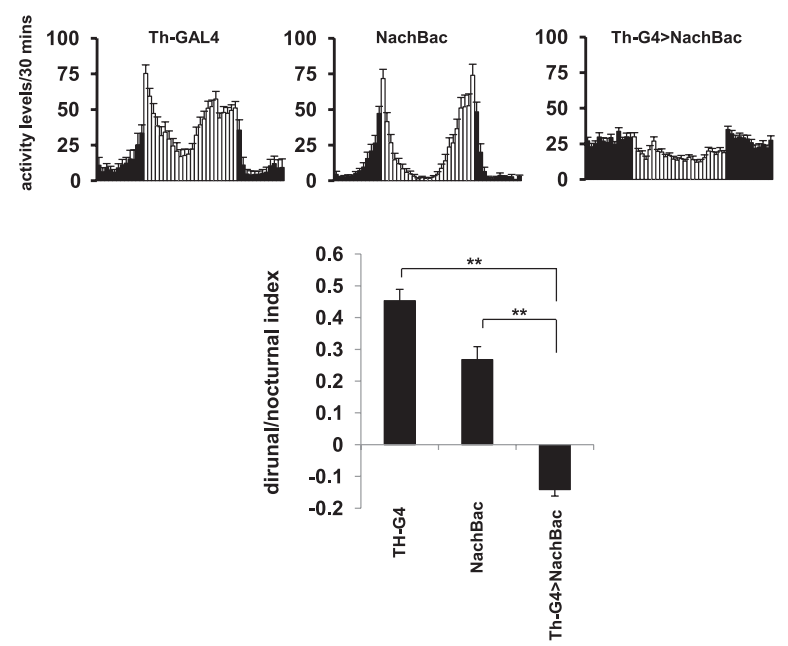

Figure 6. Increased dopamine signaling elevates CRY levels and promotes nocturnal behavior. (A) Reducing dopamine signaling decreases CRY levels in Clk ${ }^{r k}$ flies. The data show a representative Western blot of head samples from flies carrying TH-GAL4 with or without UAS-Tnt in a Clk ${ }^{\text {Irk }}$ background during light (ZT08) and dark (ZT20) phases of an LD cycle. Note that CRY levels are significantly reduced during the dark phase of the LD in the TH-GAL4>UAS-Tnt; $C l k^{r r k}$ flies as compared with $C l k^{r r k}$ mutants that do not express UAS-Tnt (cf. lanes 3 and 5). The quantification on the right depicts the average of three independent experiments. Other details are similar to those in Figures 1 and 3. $(B)$ Increasing dopamine promotes nighttime activity in wild-type flies. An artificial UAS-NachBac transgene was overexpressed in dopaminergic neurons of wild-type flies using the TH-GAL4 driver. This manipulation increased nighttime activity as compared with the TH-GAL4 driver and UAS-NachBac alone control lines. (Top panel) The average activity pattern of the indicated genotypes in LD cycles. Numbers of flies are as follows: TH-GAL4 control $(n=18)$, UAS-Nachbac control $(n=16)$, and TH-GAL4>UASNachBac $(n=20)$ in a wild-type background. (Bottom panel) Diurnal/nocturnal indices of flies for the genotypes indicated. The records are based on activity data from four consecutive 24$\mathrm{h}$ periods. Other details are similar to those in Figures 1-3. Asterisk symbols above bars denote significant differences between genotypes. $\left(^{\star}\right) P<0.05 ;\left(^{\star \star}\right) P<0.01$. Error bars indicate the SEM.

of the night, this effect was surprising, and so we wondered whether it reflected a subtle role for these molecules in acute arousal. Although arousal thresholds are higher during sleep, survival requires that animals still respond to sudden changes in the environment. To test the idea that CRY and dopamine are required for responses to sensory stimulation at night, we assayed such responses in flies mutant for CRY or dopamine. We examined sensitivity to mechanical or light stimuli during the middle of the night in wild-type Iso31, crynull $\left(\right.$ cry $\left.^{02}\right)$, cry $^{b}$, a temperature-sensitive allele of ple over its deficiency $\left[p l e^{t s 1} / D f(3 L) v n^{65 c}\right]$ (low dopamine), Clk ${ }^{I r k}$ (increased TH and CRY), and fmn (increased dopamine and CRY) flies. Specifically, we delivered a mechanical jolt or a brief light pulse at ZT20 for four consecutive nights to mutant and control flies housed within an activity monitor (see the Materials and Methods for details; Table 1; Supplemental Fig. 7A,B). Each fly's response was calculated by determining whether it showed significantly increased activity in the $30 \mathrm{~min}$ following the stimulus as compared with the prior 30 min. We found that $>80 \%$ of wild-type flies showed a positive response and $\sim 60 \%-65 \%$ of $C 1 k^{\text {Irk }}$ and $\sim 55 \%-$ $60 \%$ of $f m n$ flies showed a positive response. We believe that the slightly lower numbers for $C l k^{J r k}$ and $f m n$ reflect the very high basal activity in these flies, which sometimes made it difficult to detect a significant increase over baseline. Importantly, despite the fact that the $p l e^{t s 1} /$ $D f$ combination reduces but does not eliminate dopamine, only $31 \%$ and $21 \%$ of these mutants showed responses to light and mechanical stimuli, respectively. The magnitude of the response was also reduced (Supplemental Fig. 7A,B). The response was intact in $c^{b}{ }^{b}$ flies, suggesting that the remaining CRY in these flies, which cannot mediate circadian responses to light, is sufficient for this response. However, only $22 \%$ and $7 \%$ of $c r y{ }^{02}$ flies showed a response to light and mechanical stimuli, respectively (Table 1). In other experiments, we assayed responses of these mutants to sensory stimulation during the subjective day, and, as at night, the response of cry ${ }^{02}$ flies was reduced (data not shown). Based on these data, we propose that dopamine and CRY are required for acute arousal in response to sensory stimuli. Elevated signaling through these molecules results in nocturnal behavior

Table 1. Dopamine and CRY are required for responses to acute sensory stimulation at night

\begin{tabular}{lrrr}
\hline Genotype & Light pulse & Mechanical stimulus & Handled control \\
\hline Iso31 & $81.25 \%(16)$ & $87.5 \%(16)$ & $13.3 \%(15)$ \\
ple $^{t s 1} / D f$ & $31.25 \%(16)$ & $21.05 \%(19)$ & $11.76 \%(17)$ \\
cry $^{b}$ & $81.25 \%(16)$ & $68.75 \%(16)$ & $12.5 \%(16)$ \\
cry $^{02}$ & $22.6 \%(31)$ & $7.14 \%(28)$ & $0 \%(16)$ \\
Clk $^{r k k}$ & $58.33 \%(12)$ & $66.67 \%(15)$ & $7.69 \%(13)$ \\
fmn & $60 \%(15)$ & $53.84 \%(13)$ & $0 \%(16)$ \\
\hline
\end{tabular}

Flies that were subjected to either a mechanical jolt or light pulse at ZT20 are shown for the following genotypes: wild-type Iso31, a temperature-sensitive allele of ple over its deficiency $\left(p l e^{t s 1} /\right.$

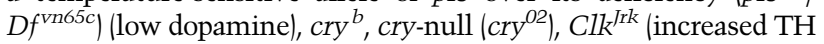
and CRY), and $f m n$ (increased dopamine and CRY) flies. A positive response was statistically determined by Student's $t$-test for each individual fly by comparing the average activity over $4 \mathrm{~d}$ exhibited in the 30-min bin after the mechanical or light stimulus with the total amount of activity in the 30-min bin before the stimulus. The handled control for the light pulse indicates flies that were removed from the incubator that were not pulsed. Shown are the percentage of flies showing a positive response to stimulus, with the number of flies tested indicated in parentheses. 
because they are normally higher at night (see the Discussion).

\section{Discussion}

We report here mechanistic insights into the switch from diurnal to nocturnal behavior in Clk mutants. In dissecting this mechanism, we also uncovered a novel role for dopamine and CRY in promoting arousal at night. We show that nocturnal behavior of Clk mutants arises, in large part, from increased dopamine signaling produced by loss of Clk regulation of $\mathrm{TH}$. The increased dopamine acts through CRY in the $1-\mathrm{LN}_{\mathrm{v}} \mathrm{s}$ to drive high activity at night (Fig. 7). This effect likely also accounts for the overall increased wake phenotype of $C 1 k^{I r k}$ mutants reported previously (Hendricks et al. 2003). We note, however, that increased wakefulness/decreased sleep is not always associated with nighttime preference for activity because sleepless (sss) mutants, which have a very dramatic reduction in sleep, are not nocturnal (Koh et al. 2008; data not shown). The nocturnal preference is specific to high dopamine because of a function of this neurotransmitter at night.

Previous studies have suggested an interaction between light and CLK, seen as increased activity of $C 1 k^{\text {Irk }}$ mutant flies following lights-off or in the dark /Allada
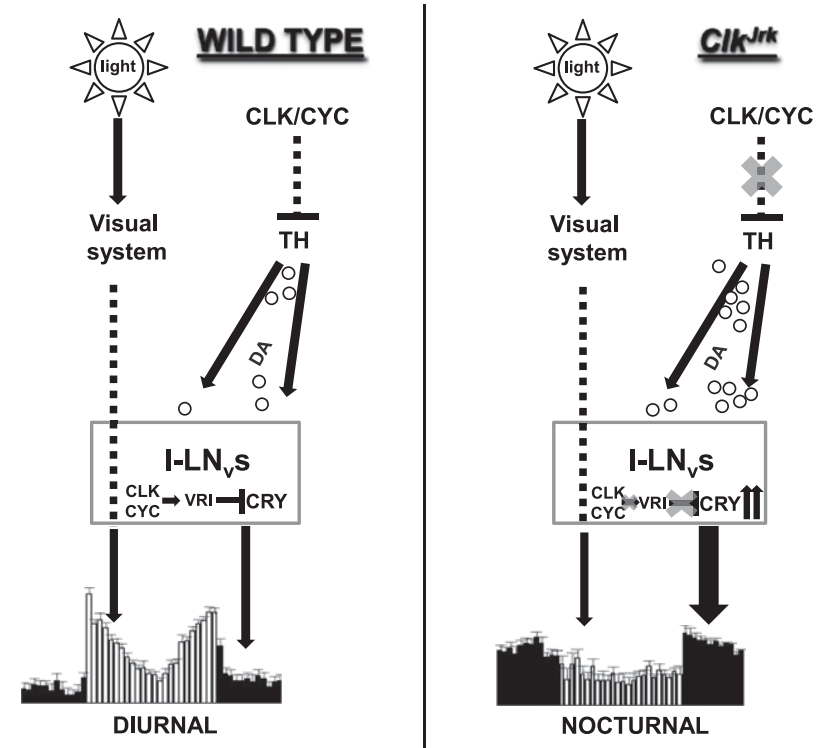

Figure 7. Role of dopamine (DA) and CRY in regulating diurnal/nocturnal behavior. $(A)$ In wild-type flies, light promotes activity during the day via $1-\mathrm{LN}_{\mathrm{v}} \mathrm{s}$. Although it has not been directly demonstrated, these effects of light on the $1-\mathrm{LN}_{\mathrm{v}} \mathrm{s}$ are likely mediated by the visual system (shown by dotted lines). In addition, CLK/CYC suppresses dopamine signaling, which would otherwise promote activity at night. CLK/CYC also suppresses CRY through dopamine as well as through its effect on vri. (B) In $C l k^{\text {Irk }}$ mutants, dopamine signaling is elevated (shown as increased extracellular dopamine), which acts through CRY to drive activity at night. As mentioned in the text, CRY may also be regulated by CLK at the transcriptional level through vrille. et al. 1998, 2003; Kim et al. 2002; Lu et al. 2008). Overexpression of CLK modulates direct effects of light on activity levels and was proposed to influence visual phototransduction pathways (Kim et al. 2002), which are required for such effects. Thus, the light-induced startle response in Drosophila is diminished or eliminated in the absence of visual and extraocular photoreception (Helfrich-Forster et al. 2001). In fact, visual mutants also show a tendency to be active at night, and we found that Clk and the visual mutants act synergistically to promote nocturnal activity (data not shown). We believe that effects of Clk and phototransduction pathways converge at the level of dopamine signaling. Light up-regulates inhibitory dopamine receptors (Shang et al. 2011), and, as shown here, Clk down-regulates dopamine synthesis. Expression of $\mathrm{TH}$ cycles in wild-type flies but is constantly high in Clk mutants, indicating that Clk is required for the daily trough in expression. Given that CLK is a transcriptional activator, its effect on $\mathrm{TH}$ is likely indirect. This is also supported by the phase of $\mathrm{TH}$ expression, which is opposite that of direct CLK targets. TH mRNA levels were also found to be high in a microarray analysis of $C l k^{\text {Irk }}$ heads (McDonald and Rosbash 2001) and, interestingly, are also elevated in mammalian Clock mutants (McClung et al. 2005). Importantly, blocking dopamine signaling, either pharmacologically or genetically, suppresses nocturnal behavior of $C l k^{I r k}$ mutants. In addition, fmn mutants have increased nighttime activity. They are not as nocturnal as $C l k^{J r k}$ flies, perhaps because the distribution of the dopamine transporter is limited (Porzgen et al. 2001); thus, most of the increased dopamine in $f m n$ may not be in the vicinity of $1-L_{\mathrm{v}} \mathrm{s}$.

We show that the nocturnal behavior of $C l k^{I r k}$ mutants is also suppressed by mutations in cry. To identify the cells required for the effect of CRY, we overexpressed CRY in the $\mathrm{Clk}^{\text {Irk }}{ }_{\text {, }}{ }{ }^{b}$ and $C l k^{\text {Irk }}{ }^{6}$ cry $^{02}$ double-mutant genetic backgrounds using various drivers for clock neurons-tim27-GAL4, Pdf-GAL4, per $^{1 b}$-GAL4 and c929GAL4-and in eyes using the gmr-GAL4 driver. Over-

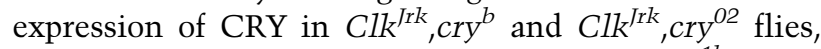
particularly in $1-\mathrm{LN}_{\mathrm{v}} \mathrm{s}$ using the $\mathrm{c} 929$ and per $^{1 b}$-GAL4 drivers, shifted the behavior of these flies toward a nocturnal preference. We were unable to phenocopy the Clk ${ }^{\text {Irk }}$ nocturnal behavior by elevating CRY levels in a wild-type background, although such manipulations increase circadian sensitivity to dim light pulses (Emery et al. 1998). CRY is necessary but not sufficient for nocturnal behavior. On the other hand, high dopamine appears to be sufficient, leading us to speculate that CRY requires input from dopamine for this phenotype (discussed below).

It is clear that the $1-\mathrm{LN}_{\mathrm{v}} \mathrm{s}$ have a prominent role in the presence of LD cycles, although perhaps not as much in DD. They are similar to s- $\mathrm{LN}_{\mathrm{v}} \mathrm{s}$ in expressing the neuropeptide PDF; however, they show a number of significant differences. In $\mathrm{LD}, 1-\mathrm{LN}_{\mathrm{v}} \mathrm{s}$ display robust oscillations of clock molecules. In fact, molecular oscillations in these neurons are phase-advanced relative to the rest of the clock network (Shafer et al. 2002; Dissel et al. 2004), but in DD, cycling in the $1-\mathrm{LN}_{\mathrm{v}} \mathrm{s}$ dampens rapidly (Yang and 
Sehgal 2001; Shafer et al. 2002). Oscillations are restored after a few cycles in DD, probably because of synchronization from other clock neurons within the clock network (Peng et al. 2003). The major role of $1-\mathrm{LN}_{\mathrm{v}} \mathrm{s}$ is in light-induced arousal, presumably through photic regulation of their electrical activity (Shang et al. 2008; Fogle et al. 2011). Photic input to $1-\mathrm{LN}_{\mathrm{v}} \mathrm{s}$ is thought to consist of a direct projection from the Hofbauer-Buchner extraretinal eyelet as well as input from the optic lobes (Helfrich-Forster 2002). It is likely that these inputs from the visual system transmit arousal-promoting stimuli to the $1-\mathrm{LN}_{\mathrm{v}} \mathrm{s}$, although this has not been directly demonstrated. Our data indicate that the arousalpromoting effect of light on $1-\mathrm{LN}_{\mathrm{v}} \mathrm{s}$ requires downregulation of dopamine signaling. This down-regulation is achieved through light induction of inhibitory dopamine receptors (Shang et al. 2011) and CLK regulation of $\mathrm{TH}$.

Both dopamine and CRY are required for acute arousal at night. An arousal-promoting role for dopamine is supported by earlier studies. The fmn mutants were shown to exhibit a decreased arousal threshold (Kume et al. 2005), whereas the ple mutants exhibit an increased arousal threshold (Riemensperger et al. 2011). We show here that this effect on arousal reflects a novel role for dopamine in sensory responses at night. CRY has not been implicated in arousal, although it promotes neural activity in a lightdependent manner. As in the case of the neural activity assay (Fogle et al. 2011), we found that arousal in response to sensory stimuli is reduced but not eliminated by the $c^{b} y^{b}$ mutant, indicating that the mechanism is distinct from the circadian response that is eliminated by $c r y^{b}$ (Stanewsky et al. 1998). Both neural activity and behavioral arousal responses are eliminated by the $c r y^{0}$ mutant (Fogle et al. 2011; present study), suggesting that the neural response underlies the behavioral effect. We propose that CRY is required at multiple levels for acute responses to sensory stimuli. In the case of circadian photoreception, it is absolutely required for phase-shifting in response to pulses of light, although not for entrainment to LD cycles (Stanewsky et al. 1998; Helfrich-Forster 2002). In the case of responses to sensory stimuli, again it is required for the startle response. Any effects of CRY on light-induced activity (physiological or behavioral) are likely to be acute, since CRY gets degraded with increased light treatment (Lin et al. 2001). Interestingly, in two different species of Bactrocera, cry mRNA levels are positively correlated with the timing of mating (An et al. 2004), which is also indicative of a regulated response required for a specific purpose. A chronic effect is seen only in the case of Drosophila Clk mutants, where levels of CRY are considerably higher than normal, and dopamine signaling is also elevated. We hypothesize that CRY only promotes nocturnal activity in flies with chronically elevated dopamine signaling because dopamine acts as a trigger to activate CRY. However, this activation may be different from activation in a circadian context, given that different mechanisms appear to underlie the circadian and arousalpromoting roles of CRY. Dopamine- and CRY-mediated locomotor activity is restricted largely to the night because of light-induced CRY degradation (Lin et al. 2001) and light-induced inhibition of dopamine signaling (Shang et al. 2011).

At night, animals sleep, and the arousal threshold is increased. However, they still need to be able to respond in case of sudden events. We speculate that dopamine and CRY are essential for this. In the case of CRY, it may arouse the animal and also reset the clock. For instance, the immediate response of an animal to a pulse of light at night is to wake up, which may be driven by the arousalpromoting role of CRY. In addition, the circadian clock must be reset, which requires the circadian function of CRY. Whether or not these roles of CRY are conserved, we speculate that dopamine functions similarly in mammals. Interestingly, melanopsin, which is the circadian photoreceptor in mammals (analogous to CRY in flies), is regulated by dopamine in intrinsically photosensitive retinal ganglion cells (ipRGCs) (Sakamoto et al. 2005). Like CRY, melanopsin is also required for acute behavioral responses to light, specifically for sleep induction in nocturnal animals during the day (Lupi et al. 2008). These ipRGCs have been proposed as functionally similar to $1-\mathrm{LN}_{\mathrm{v}} \mathrm{s}$ (Im and Taghert 2011), so a conserved function for the relevant molecules is intriguing. Finally, we note that elevated dopamine has been linked to increased nighttime activity in humans, which are, of course, diurnal like Drosophila. People with Sundown syndrome or nocturnal delirium show increased agitation and sleep disturbances in the early evening, which can be treated with anti-psychotic medications that target dopamine signaling (Falsetti 2000).

\section{Materials and methods}

\section{Drosophila stocks}

Wild-type Iso31 flies were maintained at $18^{\circ} \mathrm{C}$ or $25^{\circ} \mathrm{C}$ on a 12 -h light:dark cycle in bottles containing an agar, sucrose, water, and dried yeast medium. Clk ${ }^{\text {Irk }}$ mutants (Allada et al. 1998) and Clk deficiency Df(3L)RM5-2 flies were obtained from the Bloomington Drosophila Stock Center at Indiana University and outcrossed to an isogenic $w^{1118}$ background (Iso31) for seven generations. The molecular lesion was followed by genotyping. Most of the other fly stocks and GAL4 drivers for clock neurons are used routinely in the laboratory. gmr-GAL4, long gmr-GAL4, and $g m r$-GeneSwitch (Roman and Davis 2002) flies were obtained from the Bloomington Drosophila Stock Center. These lines were outcrossed into a common Iso31 isogenic background. The cry ${ }^{02}$ mutants were provided by Patrick Emery (University of Massachusetts Medical School). The dopamine transporter mutant fmn was provided by Rob Jackson (Tufts University). The temperaturesensitive mutant ple $e^{t s 1}$ (EMS-generated) was obtained from Ralph Hillman (Temple University) and maintained as a hemizygote over a small deficiency for the ple locus $D f(3 L) v n^{65 c}$ (Pendleton et al. 2002).

\section{Behavioral assays and statistical analyses}

Three-day-old to 5-d-old adult male flies were loaded into locomotor assay tubes containing $5 \%$ sucrose and $2 \%$ agarose and entrained to a 12 -h: 12 -h LD schedule for $4-5 \mathrm{~d}$ at $25^{\circ} \mathrm{C}$. Activity was collected in LD conditions using the Drosophila Activity 
Monitoring System (DAMS) from Trikinetics. To obtain a quantitative measure of diurnal or nocturnal behavior, the diurnal/ nocturnal index was calculated (see figure legends for details). For acute light and mechanical stimulus experiments, the Iso31 control, $c r y^{b}, c r y{ }^{02}, p^{t s 1} / D f, C l k^{I r k}$, and fmn flies (male, 2-4 d of age) were raised in LD conditions, collected, and placed in glass tubes in activity monitors. For the acute light, stimulus monitors were taken out of the incubator and briefly exposed to light $(\sim 2-3 \mathrm{sec})$ at ZT20 for four consecutive days. For mechanical stimulation, the flies were disturbed by scraping the monitors four to five times at ZT20 for four consecutive days. Control flies were neither pulsed nor aroused by mechanical stimulation but briefly taken out of the incubator. The amount of activity was calculated in 30-min bins over the $24 \mathrm{~h}$ of the LD cycle and averaged for $4 \mathrm{~d}$. For each fly, a positive response to the stimulus was assayed as a significant increase in activity $(P<0.05$, as analyzed by Student's $t$-test) in the 30 -min interval after the stimulus as compared with the $30 \mathrm{~min}$ prior to the stimulus. For all behavioral assays shown, comparisons between genotypes were made by Student's $t$-test if one variable was involved and by two-way ANOVA if two factors had to be taken into account (e.g., genotype and drug in Fig. 5B), followed by post hoc comparison using Tukey's test.

\section{Drug treatment}

Wild-type Iso31 flies and $C l k^{r r k}$ mutants were raised on normal food medium prior to the assay. Haloperidol drug (H1512) was purchased from Sigma and dissolved in DMSO and mixed in the $5 \%$ sucrose and $2 \%$ agar food medium at a final concentration of $0.0,12.5,25$, and $50 \mu \mathrm{M}$. Three-day-old to 5-d-old flies were exposed to different doses of the drug or to DMSO alone (no drug controls) for the entire duration of the locomotor assay. To determine the effect of the drug, data from days 2-5 were used for analysis.

\section{Western blot analysis}

Three-day-old to 5-d-old adult flies were entrained to 12-h:12-h LD cycles for $3 \mathrm{~d}$, and heads were collected at indicated time points for protein extraction. Western blot analysis was performed as described previously (Sathyanarayanan et al. 2004). The primary antibodies used in different assays were guinea pig anti-CLK (1:3000) (Houl et al. 2008), rabbit anti-CRY (1:3000) (Rush et al. 2006), rabbit anti-TH AB152 (1:500; Millipore), and mouse anti-HSP70 (1:5000; Sigma). Following enhanced chemiluminescence, images were obtained in a Kodak image station or from exposure to film. ImageJ software (NIH) was used for quantification of individual bands on Western blots. For all Western blot quantifications, comparisons between genotypes were made by one-way ANOVA, followed by post hoc comparison using Tukey's test.

\section{Quantitative real-time PCR}

Three-day-old to 5-d-old adult flies were maintained in a 12-h: 12-h LD cycle for $3 \mathrm{~d}$ at $25^{\circ} \mathrm{C}$ and then collected on dry ice at indicated time points on the third day of LD. Total RNA was isolated using an Ultraspec RNA isolation system (Biotecx), and cDNAs were synthesized using a high-capacity cDNA Archive kit (Applied Biosystems). Quantitative real-time PCR was performed in an ABI prism 7100 using a SYBR Green kit (Applied Biosystems). The oligos used in the assays were CNS-specific isoform ple-PA forward (5'-CAAGGCAAATGATTACGGTC-3') and ple-PA reverse (5'-GGCATTGGCCAACAAAATCT-3'), cry forward (5'-GCACACGGTGCAAATTATTGG-3') and cry re- verse (5'-TGGCGTCTTCTAGTCGAGCAT-3'), and Act5C forward ( $5^{\prime}$-ATGTCACGGACGATTTCACG- $\left.3^{\prime}\right)$ and $A c t 5 C$ reverse (5'-CGCGGTTACTCTTTCACCA-3').

\section{Acknowledgments}

We are extremely grateful to Jay Hirsh for suggesting a role of dopamine in the Clk ${ }^{\text {Irk }}$ phenotype, and to Patrick Emery for a very generous supply of anti-CRY antibodies. We thank Paul Taghert, Rob Jackson, Jeff Hall, and Ralph Hillman for fly stocks. We also thank Kyunghee Koh for helpful discussions, and Sam Zheng, Mi Shi, and Wenfeng Chen for comments on the manuscript. The work was supported by 1R01NS048471 and 1R56NS048471 grants to A.S. A.S. is an Investigator of the HHMI.

\section{References}

Allada R, White NE, So WV, Hall JC, Rosbash M. 1998. A mutant Drosophila homolog of mammalian Clock disrupts circadian rhythms and transcription of period and timeless. Cell 93: 791-804.

Allada R, Kadener S, Nandakumar N, Rosbash M. 2003. A recessive mutant of Drosophila Clock reveals a role in circadian rhythm amplitude. EMBO I 22: 3367-3375.

An X, Tebo M, Song S, Frommer M, Raphael KA. 2004. The cryptochrome (cry) gene and a mating isolation mechanism in tephritid fruit flies. Genetics 168: 2025-2036.

Blenau W, Baumann A. 2001. Molecular and pharmacological properties of insect biogenic amine receptors: Lessons from Drosophila melanogaster and Apis mellifera. Arch Insect Biochem Physiol 48: 13-38.

Cyran SA, Buchsbaum AM, Reddy KL, Lin MC, Glossop NR, Hardin PE, Young MW, Storti RV, Blau J. 2003. vrille, Pdp1, and dClock form a second feedback loop in the Drosophila circadian clock. Cell 112: 329-341.

Dissel S, Codd V, Fedic R, Garner KJ, Costa R, Kyriacou CP, Rosato E. 2004. A constitutively active cryptochrome in Drosophila melanogaster. Nat Neurosci 7: 834-840.

Emery P, So WV, Kaneko M, Hall JC, Rosbash M. 1998. CRY, a Drosophila clock and light-regulated cryptochrome, is a major contributor to circadian rhythm resetting and photosensitivity. Cell 95: 669-679.

Falsetti AE. 2000. Risperidone for control of agitation in dementia patients. Am J Health Syst Pharm 57: 862-870.

Fogle KJ, Parson KG, Dahm NA, Holmes TC. 2011. CRYPTOCHROME is a blue-light sensor that regulates neuronal firing rate. Science 331: 1409-1413.

Friggi-Grelin F, Coulom H, Meller M, Gomez D, Hirsh J, Birman S. 2003. Targeted gene expression in Drosophila dopaminergic cells using regulatory sequences from tyrosine hydroxylase. I Neurobiol 54: 618-627.

Helfrich-Forster C. 2002. The circadian system of Drosophila melanogaster and its light input pathways. Zoology (Jena) 105: 297-312.

Helfrich-Forster C, Winter C, Hofbauer A, Hall JC, Stanewsky R. 2001. The circadian clock of fruit flies is blind after elimination of all known photoreceptors. Neuron 30: 249-261.

Hendricks JC, Lu S, Kume K, Yin JC, Yang Z, Sehgal A. 2003. Gender dimorphism in the role of cycle (BMAL1) in rest, rest regulation, and longevity in Drosophila melanogaster. J Biol Rhythms 18: 12-25.

Houl JH, Ng F, Taylor P, Hardin PE. 2008. CLOCK expression identifies developing circadian oscillator neurons in the brains of Drosophila embryos. BMC Neurosci 9: 119. doi: 10.1186/1471-2202-9-119. 
Im SH, Taghert PH. 2011. Neuroscience. A CRY to rise. Science 331: 1394-1395.

Kaneko M, Hall JC. 2000. Neuroanatomy of cells expressing clock genes in Drosophila: Transgenic manipulation of the period and timeless genes to mark the perikarya of circadian pacemaker neurons and their projections. I Comp Neurol 422: 66-94.

Kim EY, Bae K, Ng FS, Glossop NR, Hardin PE, Edery I. 2002 Drosophila CLOCK protein is under posttranscriptional control and influences light-induced activity. Neuron 34: 69-81.

Koh K, Joiner WJ, Wu MN, Yue Z, Smith CJ, Sehgal A. 2008. Identification of SLEEPLESS, a sleep-promoting factor. Science 321: 372-376.

Kume K, Kume S, Park SK, Hirsh J, Jackson FR. 2005. Dopamine is a regulator of arousal in the fruit fly. J Neurosci 25: 73777384.

Lacroix A, Baldacchino V, Bourdeau I, Hamet P, Tremblay J. 2004. Cushing's syndrome variants secondary to aberrant hormone receptors. Trends Endocrinol Metab 15: 375382.

Lin FJ, Song W, Meyer-Bernstein E, Naidoo N, Sehgal A. 2001. Photic signaling by cryptochrome in the Drosophila circadian system. Mol Cell Biol 21: 7287-7294.

Lu B, Liu W, Guo F, Guo A. 2008. Circadian modulation of lightinduced locomotion responses in Drosophila melanogaster. Genes Brain Behav 7: 730-739.

Lupi D, Oster H, Thompson S, Foster RG. 2008. The acute lightinduction of sleep is mediated by OPN4-based photoreception. Nat Neurosci 11: 1068-1073.

McClung CA, Sidiropoulou K, Vitaterna M, Takahashi JS, White FJ, Cooper DC, Nestler EJ. 2005. Regulation of dopaminergic transmission and cocaine reward by the Clock gene. Proc Natl Acad Sci 102: 9377-9381.

McDonald MJ, Rosbash M. 2001. Microarray analysis and organization of circadian gene expression in Drosophila. Cell 107: $567-578$

Nitabach MN, Taghert PH. 2008. Organization of the Drosophila circadian control circuit. Curr Biol 18: R84-R93. doi: 10.1016/j.cub.2007.11.061.

Pendleton RG, Rasheed A, Sardina T, Tully T, Hillman R. 2002. Effects of tyrosine hydroxylase mutants on locomotor activity in Drosophila: A study in functional genomics. Behav Genet 32: 89-94.

Peng Y, Stoleru D, Levine JD, Hall JC, Rosbash M. 2003. Drosophila free-running rhythms require intercellular communication. PLOS Biol 1: E13. doi: 10.1371/journal.pbio. 0000013.

Porzgen P, Park SK, Hirsh J, Sonders MS, Amara SG. 2001. The antidepressant-sensitive dopamine transporter in Drosophila melanogaster: A primordial carrier for catecholamines. Mol Pharmacol 59: 83-95.

Redlin U, Mrosovsky N. 1999. Masking by light in hamsters with SCN lesions. J Comp Physiol A Neuroethol Sens Neural Behav Physiol 184: 439-448.

Riemensperger T, Isabel G, Coulom H, Neuser K, Seugnet L, Kume K, Iche-Torres M, Cassar M, Strauss R, Preat T, et al. 2011. Behavioral consequences of dopamine deficiency in the Drosophila central nervous system. Proc Natl Acad Sci 108: 834-839.

Roman G, Davis RL. 2002. Conditional expression of UAStransgenes in the adult eye with a new gene-switch vector system. Genesis 34: 127-131.

Rush BL, Murad A, Emery P, Giebultowicz JM. 2006. Ectopic CRYPTOCHROME renders TIM light sensitive in the Drosophila ovary. J Biol Rhythms 21: 272-278.
Sakamoto K, Liu C, Kasamatsu M, Pozdeyev NV, Iuvone PM, Tosini G. 2005. Dopamine regulates melanopsin mRNA expression in intrinsically photosensitive retinal ganglion cells. Eur J Neurosci 22: 3129-3136.

Sathyanarayanan S, Zheng X, Xiao R, Sehgal A. 2004. Posttranslational modification of Drosophila PERIOD protein by Protein Phosphatase 2A. Cell 116: 603-615.

Shafer OT, Rosbash M, Truman JW. 2002. Sequential nuclear accumulation of the clock proteins period and timeless in the pacemaker neurons of Drosophila melanogaster. I Neurosci 22: 5946-5954.

Shang Y, Griffith LC, Rosbash M. 2008. Light-arousal and circadian photoreception circuits intersect at the large PDF cells of the Drosophila brain. Proc Natl Acad Sci 105: 1958719594.

Shang Y, Haynes P, Pirez N, Harrington KI, Guo F, Pollack J, Hong P, Griffith LC, Rosbash M. 2011. Imaging analysis of clock neurons reveals light buffers the wake-promoting effect of dopamine. Nat Neurosci 14: 889-895.

Sheeba V, Fogle KJ, Kaneko M, Rashid S, Chou YT, Sharma VK, Holmes TC. 2008. Large ventral lateral neurons modulate arousal and sleep in Drosophila. Curr Biol 18: 1537-1545.

Smale L, Lee T, Nunez AA. 2003. Mammalian diurnality: Some facts and gaps. J Biol Rhythms 18: 356-366.

Stanewsky R, Kaneko M, Emery P, Beretta B, Wager-Smith K, Kay SA, Rosbash M, Hall JC. 1998. The cryb mutation identifies cryptochrome as a circadian photoreceptor in Drosophila. Cell 95: 681-692.

Sweeney ST, Broadie K, Keane J, Niemann H, O'Kane CJ. 1995. Targeted expression of tetanus toxin light chain in Drosophila specifically eliminates synaptic transmission and causes behavioral defects. Neuron 14: 341-351.

Taghert PH, Hewes RS, Park JH, O'Brien MA, Han M, Peck ME. 2001. Multiple amidated neuropeptides are required for normal circadian locomotor rhythms in Drosophila. J Neurosci 21: 6673-6686.

Wheeler DA, Hamblen-Coyle MJ, Dushay MS, Hall JC. 1993. Behavior in light-dark cycles of Drosophila mutants that are arrhythmic, blind, or both. J Biol Rhythms 8: 67-94.

White BH, Osterwalder TP, Yoon KS, Joiner WJ, Whim MD, Kaczmarek LK, Keshishian H. 2001. Targeted attenuation of electrical activity in Drosophila using a genetically modified $\mathrm{K}^{+}$channel. Neuron 31: 699-711.

Yang Z, Sehgal A. 2001. Role of molecular oscillations in generating behavioral rhythms in Drosophila. Neuron 29: 453-467.

Zheng X, Sehgal A. 2008. Probing the relative importance of molecular oscillations in the circadian clock. Genetics 178: $1147-1155$. 


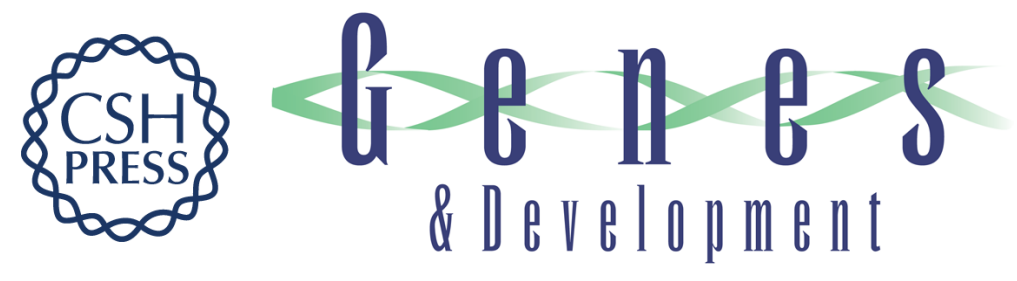

\section{Dopamine acts through Cryptochrome to promote acute arousal in Drosophila}

Shailesh Kumar, Dechun Chen and Amita Sehgal

Genes Dev. 2012, 26: originally published online May 11, 2012

Access the most recent version at doi:10.1101/gad.186338.111

\section{Supplemental http://genesdev.cshlp.org/content/suppl/2012/05/09/gad.186338.111.DC1 Material}

References This article cites 47 articles, 12 of which can be accessed free at: http://genesdev.cshlp.org/content/26/11/1224.full.html\#ref-list-1

\section{License}

Email Alerting

Receive free email alerts when new articles cite this article - sign up in the box at the top Service 